\title{
Research on Denoising Method of Ice Thickness Model of Transmission Line Based on Gauss Distribution Model
}

\author{
Weiwei Yang ${ }^{1, a}$, Xuemin $\mathrm{An}^{1, \mathrm{~b}}$, Min Zhang ${ }^{1, \mathrm{c}}$, Huiping Zheng ${ }^{1, \mathrm{~d}}$ and Qi Yang ${ }^{1, \mathrm{e}}$ \\ ${ }^{1}$ State Grid Shanxi Electric Power Research Institute, Taiyuan, 030001, China;

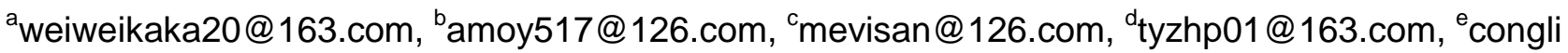 \\ nyangqi@sina.com
}

Keywords: Gauss Distribution; Denoising; Ice Thickness

\begin{abstract}
In order to predict the ice thickness of transmission line under future's meteorological factors, this paper puts forward a denoising method of ice thickness model of transmission line based on Gauss distribution model, with which an ice thickness model is built, and then the simulation graphics and performance of experimental data before and after denoising are compared. By analysis, it is proved that there is better denoising performance and more accurate simulation results in the process of denoising with Gauss distribution model, which can optimize the data denoising of icing thickness model of transmission line.
\end{abstract}

\section{Introduction}

The original data is obtained from each practical application system. For lack of a unified standard definition of the data from each application system, there is also a big difference between data structure, which results in random data. Data preprocessing refers to data handling before the main process, which will be disturbed by various noises among the data collected by online ice monitoring system. In order to improve the quality of the original data, it is necessary to take denoising pretreatment on them by various methods such as data integration, data cleaning and data transformation, etc. With all these methods used, the original data can be greatly improved before using the data application, which can significantly enhance the accuracy of the model.

\section{The mechanism of ice formation and the influencing factors}

\section{The mechanism of wire ice formation}

Cold rain splashed down the objects in the temperature below freezing $\left(0^{\circ} \mathrm{C}\right)$ to form ice, if condensation on the wire, it is wire ice. Ice formation conditions: First of all, it must be the freezing temperature $\left(<0^{\circ} \mathrm{C}\right)$; The second, it must have enough high humidity ( $\left.\mathrm{RH} \%>85 \%\right)$; Once again, there are wind speed to ensure themovement ofwater droplets in the air ( $>1 \mathrm{~m} / \mathrm{s})$.

Based on the ice to the harm of power grid, transmission line icing is divided into five categories: glaze, mixed battle (hard rime), rime, snow and frost Song (soft rime).

1) Glaze is pure, hard, and transparent ice in nature, and density of which is 0.8 to $0.9 \mathrm{~g} / \mathrm{cm}^{3}$ or higher, whose adhesive force is very strong. Glaze is generally formed at low altitudes, by cooling the rain or drizzle down in formed on the object below the freezing temperature, the temperature is -2 to $0^{\circ} \mathrm{C}$, it is most harmful to power lines.

2) Mixed battle of nature are opaque (cream) or transparent ice, often forms by the opaque and transparent ice crisscross, density of which 0.6 to $0.8 \mathrm{~g} / \mathrm{cm}^{3}$. Mixed battle general forms in the lowland, from the cloud to the ground of the ice crystals or having rain fog, the temperature of which is -5 to $0^{\circ} \mathrm{C}$, Hard and strong adhesive force. The harm for transmission line is second only to Glaze.

3) Rime is a white or milky and opaque granular deposit of ice, the density of which is 0.3 to $0.6 \mathrm{~g} / \mathrm{cm}^{3}$, its adhesion strength is quite weak, the temperature is -13 to $7^{\circ} \mathrm{C}$.

4) Snow in the lowlands is dry, low density, weak adhesion force. For condensation in hills is snow and sleet or fog, weight, density of which is 0.1 to $0.3 \mathrm{~g} / \mathrm{cm}^{3}$. After a lot of snow melt and 
frozen, Adhesion becomes a mixture of snow and ice,which can achieve very high weight and volume.

5) Soft rime is White, snowy, irregular needle crystal, very crisp and light, weaker adhesion force. The density of which is 0.05 to $0.3 \mathrm{~g} / \mathrm{cm}^{3}$, Generally is by the water vapor condenses directly from in the air, in the calm and cold weather, the temperature is below $-10^{\circ} \mathrm{C}$.

\section{The influencing factors of wire ice}

There are many factors that can influence the wire ice, mainly about meteorological factors, terrain and geographical conditions, altitude and wire suspension height.

1) Meteorological factors: mainly include air humidity, environmental temperature, wind speed, wind direction, the diameter of the cooling water droplets or fog droplets in the air, condensation level.

2) The terrain and geographical environment: mountains, slope direction and watershed also significantly influence the ice.

3) Altitude and wire suspension height: The higher thealtitude, the easier to ice, and theice thickness is bigger.

\section{Acquisition of the data}

The selected data of this article is derived from the ice online detection system, the system is installed in the tower 109 of $220 \mathrm{kV}$ transmission line located in the leeward slope of a certain area type mountain which is watershed topography. Due to the line crossing watershed and open space, it's easy to form strong winds and severe ice conditions, especially in the top of the mountain and the windward slope, under the action of wind, the air mass containing a cooling water is forced up along the mountainside and then adiabatic expansion, what makes the cooling water content and the wire ice increase, with this system, it can collect the change of the weight of the transmission wire and the slope Angle of the insulator string and so on, measure the environmental temperature and humidity, wind speed, wind and other meteorological information; and Get all sorts of relevant data parameters. Packaging the preliminary data, with the help of GSM wireless network to the monitoring center, it can calculate the thickness of ice at the query terminal using mechanics formula.

\section{Denoising algorithm based on Gauss distribution model}

\section{Basic Idea}

The center of the data can be obtained from contracted data through Gauss distribution model until it is smaller than a certain error. Each step is fitted through normal distribution and obtains the variance; points beyond the variance are removed in proportion until convergence.

\section{Specific Content}

1) The original data is divided into $\mathrm{N}$ equal intervals on the $\mathrm{X}$ axis and data distribution of each interval can be recognized as a normal distribution model; the mean value $\mu$ and standard deviation $\sigma$ of the normal distribution can be obtained. Influenced by a large amount of noise data, the mean value $\mu$ and standard deviation $\sigma$ obtained above are not the most ideal values. As a result of that, the rest of the data shall be undertaken an iterated operation.

$$
\begin{gathered}
f(x)=\frac{1}{\sqrt{2 \pi} \sigma} \sqrt{-\frac{(x-\mu)^{2}}{2 \sigma^{2}}} \\
\mu=\left(\sum_{i=1}^{n} x_{i}\right) / n \\
\sigma=\sqrt{\sum_{i=1}^{n}\left(x_{i}-\mu\right)^{2} / n}
\end{gathered}
$$

The larger the value $\sigma$ is, the more dispersed the data distributes, and the data is apt to 
distribute near value $\mu$. Data in each interval should be undertaken finite iterations until a graduate convergence of $\sigma$.

2) After the operation of each interval, an ideal error parameter should be set within the allowable error range according to the requirements of data mining, so that the data obtained is within the ideal error range.

The ideal error parameters initialized is $\mathrm{m} \%$. Random sampling 20 data from each interval as a small normal distribution model; the mean value $\mu$ and standard deviation $\sigma$ of the random sample can be obtained by the same operation. Compare the mean value $\mu$ and standard deviation $\sigma$ between the random sample and the whole interval: if the error is within $\mathrm{m} \%$, the denoising of this interval turns out to be successful; if the error is beyond $m \%$, there should be an iterative operation on all the data in the interval until the error is within the $\mathrm{m} \%$ or the maximum number of iteration is reached.

3) Finally after all the data in each interval is handled, a distribution curve is fitted with Matlab, and data shall be further processed and model be established.

\section{Ice Thickness Model of Transmission Line Based on Gauss Distribution Model}

This paper researches mainly on ice thickness model of transmission line based on Gauss distribution model. It uses the monitoring system of meteorological data of ice on a certain pole of a transmission line, with Matlab program as well as Gauss distribution model, it aims at taking on a pretreatment on the data in order to obtain the ice thickness model of the transmission line.

\section{Establishment of ice thickness model of transmission line based on Gauss distribution model}

Firstly, process the raw data according to the first step of the improved algorithm, which the $\mathrm{X}$ axis is divided into $\mathrm{N}$ equal parts; and then take preprocessing on the data of each interval so as to obtain the mean value and variance. All noise points beyond error shall be removed, and finally the data after processing shall be imported into Matlab for normalization processing; when the model is built, it is used to test the sample for accuracy of the model. Figure 1 and figure 2 show respectively the simulation results of noise-free pretreatment and the ice thickness model of transmission line of denoising pretreatment based on Gauss distribution model.

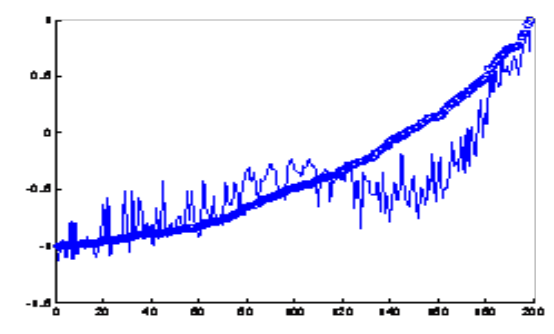

(a) Training fitting curve

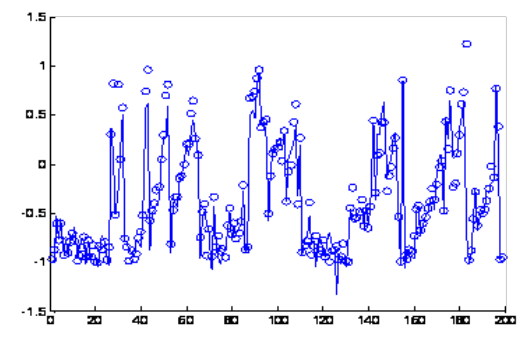

(b) Test fitting curve

Fig.1. Simulation curves of ice thickness model of transmission line with noise-free pretreatment

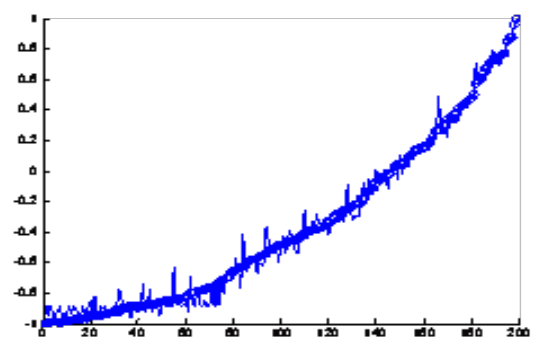

(a) Training fitting curve

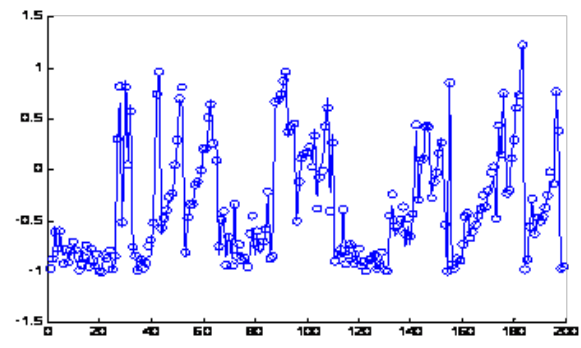

(b) Test fitting curve

Fig.2. Simulation curves of ice thickness model of transmission line based on Gauss distribution model 


\section{Comparison of simulation results}

Figure 1 shows simulation curves of ice thickness model of transmission line with noise-free pretreatment. Figure 1(a) and 1(b) are respectively the simulation training fitting curve and test fitting curve of this model, from which we can see that it is not ideal of the fitting result for both training sample to practical training curve as well as test sample curve to practical test curve. There exists a considerable amount of discrete points.

Figure 2 shows simulation curves of ice thickness model of transmission line based on Gauss distribution model. Figure 2(a) and 2(b) are respectively the simulation training fitting curve and test fitting curve of this model, from which we can see a good fitting result for both training sample to practical training curve as well as test sample curve to practical test curve. There is almost no deviation discrete point exists.

We can conclude from the figures that compared with the simulation curves of ice thickness model of transmission line with noise-free pretreatment, there is a higher fitting result on both training sample to practical training curve as well as test sample curve to practical test curve of the simulation curves to ice thickness model of transmission line based on Gauss distribution model.

A comparison of the identification results between various performance indicators of two models are shown in Table 1.

Tab 1 Comparison table of performance indexes of models

\begin{tabular}{|c|c|c|}
\hline Performance Index & $\begin{array}{c}\text { Noise-free Pretreatment } \\
\text { Model }\end{array}$ & $\begin{array}{c}\text { Pretreatment Model based on } \\
\text { Gauss Distribution }\end{array}$ \\
\hline Training Error & 0.0040302 & 0.0020981 \\
\hline SSE & 0.7001 & 0.0020 \\
\hline MSE & 0.0051 & 0.0001 \\
\hline
\end{tabular}

The calculation of error parameter SSE (sum variance) is the sum of square of the corresponding points' error of the fitting data and the original data; the closer the SSE value is to 0, the better selection and fitting result is of the model, and the more successful the data prediction is. The value of MSE (average variance) is the average value of the sum of square of the corresponding points' error of the fitting data and the original data, that is SSE/n; there is no big difference between SSE/n and SSE, which also means that the closer the SSE value is to 0, the better selection and fitting result is of the model and the more successful the data prediction is. In the Table 1, the SSE of noise-free preprocessing model is 0.7001, MSE is 0.0051, while the two values of preprocessing model based on Gauss distribution are 0.0020 and 0.0001 , in which the two error parameters are both close to 0 ; however it is much closer to 0 that the value of preprocessing model based on Gauss distribution is. Besides, the training error of preprocessing model based on Gauss distribution is 0.0040302 while the noise-free preprocessing model is 0.0020981 . After a comparison of the three error performance parameters, following can be concluded: the simulation ice thickness can be more accurately identified by preprocessing model based on Gauss distribution than noise-free preprocessing model.

From the comparison of the simulation figures of figure 1 and 2 as well as the related performance indexes in table 1, we can make the conclusion: the simulation ice thickness can be more accurately identified by preprocessing model based on Gauss distribution than noise-free preprocessing model.

\section{Conclusion}

The normal distribution model proposed in this paper undertakes a denoising pretreatment on the original data by simple division of the interval and data iteration, from which the data that has an impact on the quality of the data is removed in order to obtain a more accurate original data of the model. Through a comparison between the noise-free preprocessing model and a simulation figure of preprocessing model based on Gauss distribution, this experiment verifies the feasibility, correctness and accuracy of the model. The experimental result shows that the simulation ice thickness can be more accurately identified by preprocessing model based on Gauss distribution. 


\section{References}

[1] T.Liu, H.GaoData. Denoising algorithm based on Gauss distribution model[J]. China New Telecommunications, 2015 (17) 110-111.

[2] L.Fang, P.Zhang. Overveiw on some arithmetics for image denoising[J]. Industrial Control Computer, 2010 (23) 73-74.

[3] D.F.Liu, W.H.Liao, N.Dai. Research and implementation for denoising noisy scattered point data[J]. Journal of Southeast University (Natural Science Edition), 2007 (37) 1108-1112.

[4] Y.Zhang, X.M.Liu, Y.Sui, Y.Guan. Research and implementation of denoising method based on K-neighbors[J]. Journal of Computer Applications, 2009 (29) 1011-1014.

[5] X.M.Long, J.Zhou. Image-denosing method based on mixture Gaussian models[J]. Computer Engineering and Applications,2005 (21) 100-103. 